\title{
IKERLANAK
}

\section{ASYMMETRIC FLOW NETWORKS}

by

Norma Olaizola and Federico Valenciano

2012

Working Paper Series: IL. 60/12

Departamento de Fundamentos del Análisis Económico I

Ekonomi Analisiaren Oinarriak I Saila

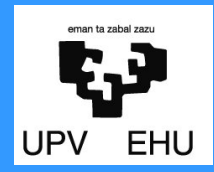

University of the Basque Country 


\title{
Asymmetric flow networks*
}

\author{
By Norma Olaizola ${ }^{\dagger}$ and Federico Valenciano ${ }^{\ddagger}$
}

June 22, 2012

\begin{abstract}
This paper provides a new model of network formation that bridges the gap between the two benchmark models by Bala and Goyal, the one-way flow model, and the two-way flow model, and includes both as particular extreme cases. As in both benchmark models, in what we call an "asymmetric flow" network a link can be initiated unilaterally by any player with any other, and the flow through a link towards the player who supports it is perfect. Unlike those models, in the opposite direction there is friction or decay. When this decay is complete there is no flow and this corresponds to the one-way flow model. The limit case when the decay in the opposite direction (and asymmetry) disappears, corresponds to the two-way flow model. We characterize stable and strictly stable architectures for the whole range of parameters of this "intermediate" and more general model. We also prove the convergence of Bala and Goyal's dynamic model in this context.

JEL Classification Numbers: A14, C72, D20, J00

Key words: Non-cooperative network formation, Asymmetric flow, Stability, Efficiency, Dynamics.
\end{abstract}

${ }^{*}$ We thank Coralio Ballester and Francesco Feri for helpful comments. Of course, the usual disclaimer applies. This research is supported by the Spanish Ministerio de Economía y Competitividad under projects ECO2009-11213 and ECO2009-07939, co-funded by the ERDF. Both authors also benefit from the Basque Government's funding to Grupos Consolidados GIC07/146-IT-377-07 and GIC07/22-IT-223-07.

${ }^{\dagger}$ BRiDGE group (http://www.bridgebilbao.es), Departamento de Fundamentos del Análisis Económico I, Universidad del País Vasco UPV/EHU, Avenida Lehendakari Aguirre 83, 48015 Bilbao, Spain; norma.olaizola@ehu.es.

${ }^{\ddagger}$ BRiDGE group (http://www.bridgebilbao.es), Departamento de Economía Aplicada IV, Universidad del País Vasco UPV/EHU, Avenida Lehendakari Aguirre 83, 48015 Bilbao, Spain; federico.valenciano@ehu.es. 


\section{Introduction}

The seminal paper by Bala and Goyal (2000a) introduced two benchmark models of network formation: the "one-way flow" model, and the "two-way flow" model. In both models each player can unilaterally create (i.e. initiate and support) links with any other player. ${ }^{1}$ These models differ in the way that the information, or other benefits ${ }^{2}$ flow through the resulting network. In the one-way flow model the flow through a link runs only towards the player that supports it, while in the two-way flow model the flow through a link runs in both directions irrespective of who supports it. It is not surprising that such different models yield very different conclusions. In the absence of friction or decay, the stable (in the sense of Nash equilibrium) networks are merely those minimally connected in either model, which means completely different architectures in either case. The situation is also completely different in regard to stability in the strict Nash sense (i.e. where any unilateral deviation of any player means a loss): in the one-way flow model the "wheel" is the only strictly stable architecture, while in the two-way flow model the only strictly stable architecture is the "center-sponsored star". In the presence of decay things become more complicated, but remain very different from one model to the other.

Both models have been extended in several directions, but those extensions take one model or the other as starting point or reference model and ignore the other. ${ }^{3}$ To the best of our knowledge, no joint generalization has been provided so far, that is, no model has been proposed that integrates both models as particular cases. Notwithstanding, these two benchmark models look like extreme cases in an unspecified sense of an unspecified model, so different that comparison is difficult given the lack of intermediate models. One may, for instance, wonder about how the "transition" from wheels to stars occurs, but no transition is possible without intermediate situations or models. This paper provides such an "intermediate" model: a model of network formation that in fact includes as particular extreme cases the two Bala and Goyal benchmark models.

As in those two benchmark models, in what we call an "asymmetric flow" network a link can be initiated unilaterally by any player with any other. As in the one-way flow model (without decay), the flow towards the player who initiates a link is perfect, without no friction or decay, but in the opposite direction it is not. More precisely, if player $i$ supports a link with $j$ but player $j$ does not support a link with $i$, all the

\footnotetext{
${ }^{1}$ In Jackson and Wolinsky's (1996) benchmark model the formation of a link between two players requires the agreement of both.

${ }^{2}$ Although other interpretations are possible, we always interpret the flow in terms of information.

${ }^{3}$ The two-way flow model has received more attention, see e.g. Bala and Goyal (2000b), Goyal and Vega-Redondo (2005), Galeotti et al. (2006), McBride (2006), Feri (2007), Hojman and Szeidl (2008), Bloch and Dutta (2009). For extensions of the one-way flow model see Galeotti (2006), Billand et al. (2008), Derks et al. (2009), Derks and Tennekes (2009). We also address separately the extension of either model in the presence of constraints (Olaizola and Valenciano, 2011, 2012). Excellent books surveying this literature are Goyal (2007), Jackson (2008) and Vega-Redondo (2007). See also Jackson's (2010) survey.
} 
information at node $j$ reaches $i$ without friction through this link, but only a fraction $\alpha$ of the information at node $i$ reaches $j$ through this link. Note that when $\alpha=0$ this is the one-way flow model, while when $\alpha=1$ the asymmetry of flow disappears and this is the two-way flow model. In order to cover it too we include $\alpha=1$ in our study as a limit case. A second parameter of the model is the cost $c$ (which we assume to be homogeneous across players) of initiating a link. Thus the two benchmark models are actually the extreme cases of this asymmetric flow model.

This new model has inherent interest in itself, apart from the nice fact of including both benchmark models as extreme particular cases. First, it is worth noting that asymmetry actually occurs in many contexts. In many situations the flow of information between two agents is not equally fluent in both directions, thus taking to some extent characteristics from both models. For instance, in an interchange between a boss and a subordinate where the boss is the only one supposed to receive information (one-way flow) it is reasonable to expect that some information may also flow in the opposite direction (e.g. about the mood of the boss). Another example is communication between two people in a language in which they are differently fluent. ${ }^{4}$ Asymmetry appears also in telecommunications, where within the bandwidth of a system data speed or quantity differs from one direction to the other direction. In all these cases one is in the presence of two-way asymmetric flow (or "asymmetric flow" for short). ${ }^{5}$

This model raises questions about stable, strictly stable and efficient architectures and the answers go beyond the dichotomy of "wheels versus stars". Here again wheels and center-sponsored stars are encountered (for certain configurations of values of the two parameters, $\alpha$ and $c$ ), but so are new richer structures such as oriented trees, with the line as an extreme case among them, and other more complex architectures. We study the ranges for the parameters where such architectures are strictly stable, which of them overlap and where (in particular this may be the case for wheels and stars for certain ranges of these parameters). In fact all these structures, including the wheel and the center-sponsored star, turn out to be particular cases of a general architecture precisely described and characterized as the only one for which strict stability may hold. A similar study about non-strict stability yields a characterization of the architecture of Nash networks. We also study the efficiency of these architectures and see how in general stability and efficiency do not go together.

Finally, we address the extension in this setting of Bala and Goyal's dynamic model and results. This extension is achieved at the cost of a lengthy algorithmic constructive proof of the existence of a sequence of best responses which, starting from an arbitrary network, yields a strict Nash network.

A brief discussion of the conclusions of stability for the simplest case, a three-player

\footnotetext{
${ }^{4}$ Including formal languages, such as mathematical formalism in different fields or programming languages.

${ }^{5}$ To our surprise, entering the term "asymmetric flow" in Google yielded a large number of scientific works in physics, informatics, medicine or sociology. In informatics the term "two-way flow networks" appears in regard to these situations.
} 

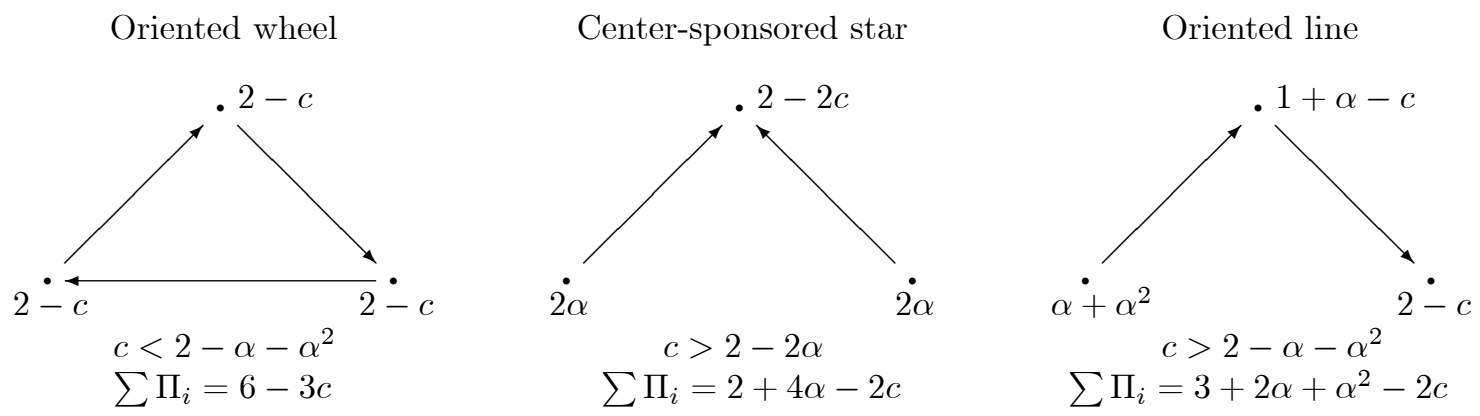

Figure 1: Strict Nash networks for $n=3$

society, provides an easy advance illustration of the general model and results presented and proved in subsequent sections. Consider a three-player society and assume that (i) each node has an information of value 1 for the other players; (ii) information flows through a link without loss in the direction of the player that supports it, but only a fraction $\alpha(0 \leq \alpha \leq 1)$ flows in the opposite direction; (iii) the cost of initiating a link is $c(0<c<1)$. It can easily be checked that the only architectures that can be strict Nash are the oriented wheel, the center-sponsored star and the oriented line (each of them for a certain range of the parameters $\alpha$ and $c$ ). These three structures are represented in Figure 1 along with the payoff of each player, the condition for strict stability of each structure and the aggregate payoff. ${ }^{6}$ Figure 2 shows the regions where each of these three architectures is a strict Nash network. When $c<2-2 \alpha$ only the wheel is strictly stable, but beyond this straight line, i.e. when $c>2-2 \alpha$, the center-sponsored star is also strictly stable. This situation persists as long as $c<2-\alpha-\alpha^{2}$, but when $c=2-\alpha-\alpha^{2}$ a player in a wheel is indifferent between keeping or deleting her/his link, that is to say both structures wheel and line are stable but not strictly stable. Beyond this point, when $c>2-\alpha-\alpha^{2}$ the line is strict Nash but the wheel is not. In short, parabola $c=2-\alpha-\alpha^{2}$ divides the region of possible values of the parameters into two disjoint regions: below it the wheel is strict Nash but the line is not, and above it the line is strict Nash but the wheel is not. The third structure, the center-sponsored star, is strictly stable above the straight line $c=2-2 \alpha$, thus sharing strict stability first with the wheel and then with the line. Finally, as mentioned previously, it is easy to check that no other architecture is strict Nash for any value of the parameters. Note also that the points with the form $(0, c)$ in the rectangle represented correspond to the one-way flow model, where the only strict Nash architecture is the wheel, and those with the form $(1, c)$ correspond to the two-way flow model, where the only strict Nash architecture is the center-sponsored star.

As to non-strict Nash stability, the only architecture apart from the three considered above that can be stable consists of four links, where one player links with the other

\footnotetext{
${ }^{6}$ As in all figures, nodes are represented by dots (without labels unless convenient), and links by arrows between them with the convention that the node at the tip of the arrow supports it.
} 


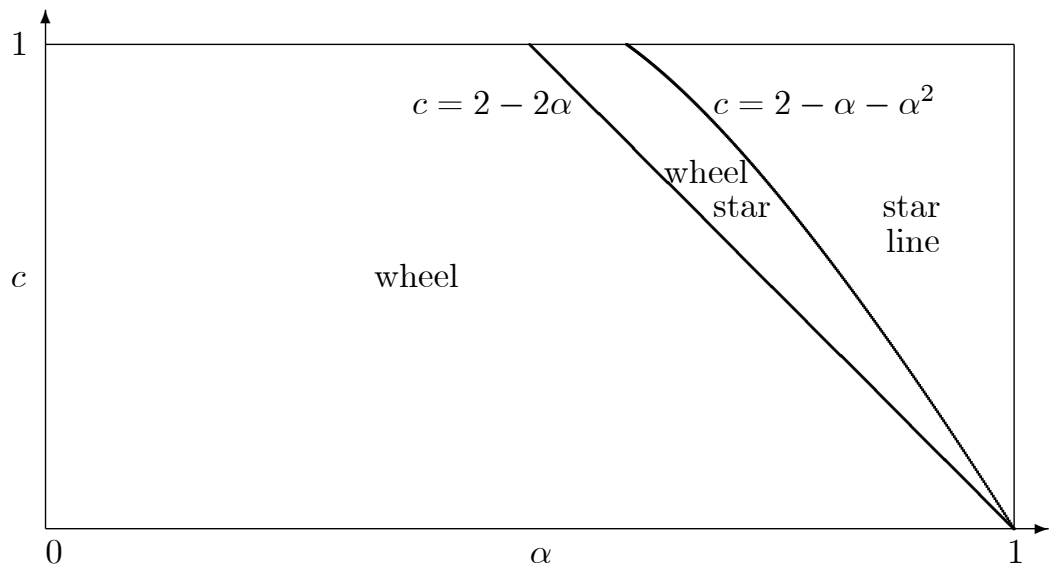

Figure 2: Ranges of strict stability for $n=3$

two and $\mathrm{s} /$ he is linked by both. This structure is stable for $c \leq 1-\alpha$.

Now consider efficiency in the sense of aggregate payoff. The following conclusions may be reached for the three possible architectures of a strict Nash network. The aggregate payoff of the wheel is $W=6-3 c$, that of the center-sponsored star is $S=2+4 \alpha-2 c$, and that of the line is $L=3+2 \alpha+\alpha^{2}-2 c$. Which of these networks is most efficient depends on the parameters. As represented in Figure 3, the rectangle containing all possible pairs $(\alpha, c)$ is divided into three regions by the parabola $c=3-2 \alpha-\alpha^{2}$ and the straight line $c=4-4 \alpha$ :

- below the parabola: $W>L>S$,

- between the parabola and the straight line: $L>W>S$,

- above the straight line, as long as $\alpha<1: L>S>W$.

Moreover, it can easily be checked that in the first region the wheel is more efficient than any other network, while in the other two regions it is the oriented line. But observe that in view of the analysis of strict stability, in the region between parabolas $c=2-\alpha-\alpha^{2}$ (dotted line in Figure 3) and $c=3-2 \alpha-\alpha^{2}$, although the wheel is efficient it is not strictly stable. Note also that for $\alpha=1$ both the line and the star are efficient but only the latter is strictly stable.

As the reader may guess, things become more complex when the number of players increases. Nevertheless, we show that things remain tractable and results extend in a smooth and clear way. In particular, oriented wheels and root-oriented trees are stable for certain ranges of values of the parameters, ${ }^{7}$ but other more complex structures appear as Nash and as strict Nash networks for certain values of the parameters, though they are all particular cases of a general pattern.

The rest of the paper is organized as follows. Section 2 outlines the basic model

\footnotetext{
${ }^{7}$ Observe that for the three-player case the center-sponsored star and the oriented line are the only root-oriented trees, while in general they are only the two extreme cases: the shortest and the longest oriented trees.
} 


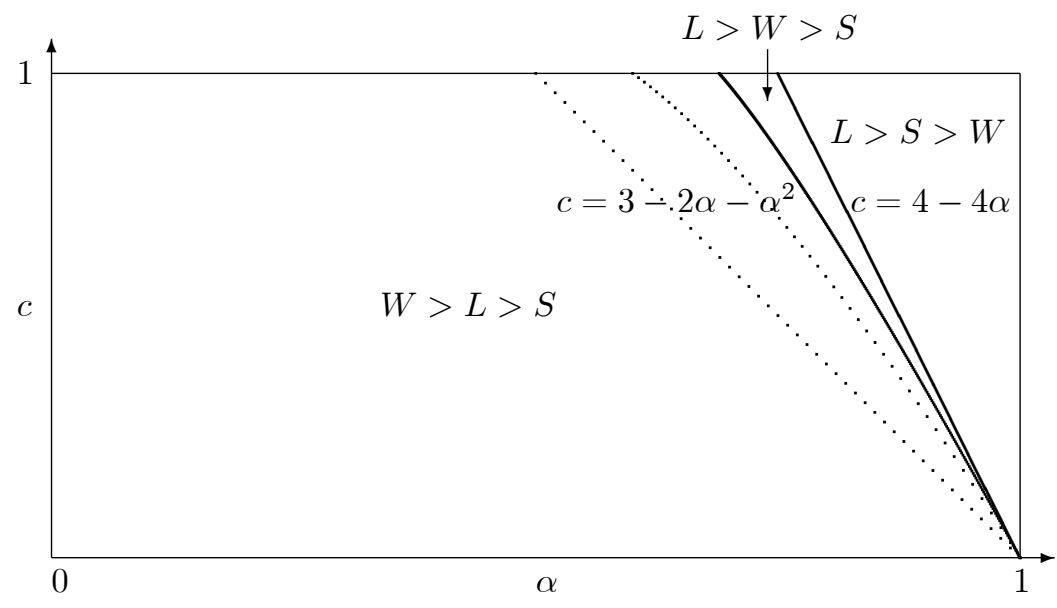

Figure 3: Ranges of efficiency for $n=3$

and gives the necessary notation and terminology. Section 3 studies stability and strict stability. Efficiency is dealt with in Section 4. Section 5 applies Bala and Goyal's dynamic model to this setting. Finally, Section 6 summarizes the main conclusions.

\section{The asymmetric flow model}

Let $N=\{1,2, . ., n\}$ denote the set of nodes or players. Each player may initiate and support links with other players. Let $g_{i j} \in\{0,1\}$ denote the existence $\left(g_{i j}=1\right)$ or not $\left(g_{i j}=0\right)$ of a link connecting $i$ and $j$ initiated by $i$. When such a link exists it is referred to as "link $\overleftarrow{i j}$ ". It is assumed that a link $\overleftarrow{i j}$ allows the transmission of information or other benefits without friction from $j$ to $i$, but with decay $\alpha(0 \leq \alpha \leq 1)$ from $i$ to $j$. Vector $g_{i}=\left(g_{i j}\right)_{j \in N \backslash\{i\}} \in\{0,1\}^{N \backslash\{i\}}$ specifies the links supported by $i$ and is referred to as a strategy of player $i . G_{i}:=\{0,1\}^{N \backslash\{i\}}$ denotes the set of $i$ 's strategies and $G_{N}=G_{1} \times G_{2} \times \ldots \times G_{n}$ the set of strategy profiles. A strategy profile $g \in G_{N}$ determines a directed $N$-network $\left(N, \Gamma_{g}\right)$, where

$$
\Gamma_{g}:=\left\{(i, j) \in N \times N: g_{i j}=1\right\}
$$

which is identified with $g$ and referred to as network $g$. If $M \subseteq N$ then $\left.g\right|_{M}$ denotes the $M$-network $\left(M, \Gamma_{\left.g\right|_{M}}\right)$ with

$$
\Gamma_{\left.g\right|_{M}}:=\left\{(i, j) \in M \times M: g_{i j}=1\right\},
$$

which we refer to as the $M$-subnetwork of $g$. As $N$ is usually clear from the context, we generally write just "network" instead of " $N$-network".

Given a network $g, \bar{g}_{i j}:=\max \left\{g_{i j}, g_{j i}\right\}$ defines the non-directed network $\bar{g}$ whose links represent the existence of communication, perfect or otherwise but direct, between nodes in $g$. A path of length $k$ from $j$ to $i$ in $g$ is a sequence of $k+1$ players $j_{0}, j_{1}, . ., j_{k}$, 
s.t. $j=j_{0}, i=j_{k}$, and for all $l=1, . ., k, \bar{g}_{j_{l-1} j_{l}}=1$. A reversed link on such a path is a pair $j_{l-1} j_{l}$ in the path s.t. $g_{j_{l} j_{l-1}}=0$. If the path does not contain any reversed links it is called $i$-oriented and written as $i \stackrel{g}{\longleftarrow} j$. The distance between two nodes is the length of the shortest path connecting them. The discounted length of a path from $j$ to $i$ in $g$ is defined as follows: 0 if it is $i$-oriented or $\alpha=1$; the number of reversed links on the path if $\alpha>0$; and $\infty$ if $\alpha=0$ and there is at least one reversed link.

It is assumed that each node contains a particular type of information valuable to other players, and as a link $\overleftarrow{i j}$ transmits information from $j$ to $i$ without friction but with decay $\alpha$ from $i$ to $j$, the best communication channel from node $j$ to $i$ is not the shortest path but the path with the smallest discounted length. This motivates the following definition.

Definition 1 The "discounted distance" from node $j$ to node $i(i \neq j)$ in a network $g$, denoted by $\delta(i, j ; g)$, is the discounted length of the path from $j$ to $i$ for which the discounted length is minimal. If no path between $i$ and $j$ exists we write $\delta(i, j ; g)=\infty$, and we set $\delta(i, i ; g)=0$.

Thus, if there is an $i$-oriented path from $j$ to $i$ then $\delta(i, j ; g)=0$. Note that the discounted distance is not symmetric, i.e. it may well be the case that $\delta(i, j ; g) \neq$ $\delta(j, i ; g) .^{8}$ In Figure 4 an example illustrates the notion of discounted distance.
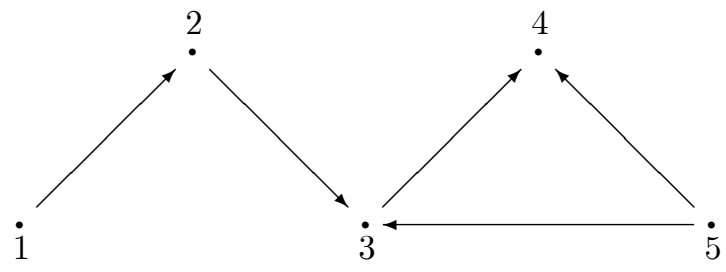

$$
\begin{array}{llll}
\delta(1,2)=1 & \delta(4,1)=0 & \delta(2,4)=2 & \delta(4,3)=0 \\
\delta(2,1)=0 & \delta(1,5)=2 & \delta(4,2)=0 & \delta(3,5)=0 \\
\delta(1,3)=2 & \delta(5,1)=1 & \delta(2,5)=1 & \delta(5,3)=1 \\
\delta(3,1)=0 & \delta(2,3)=1 & \delta(5,2)=1 & \delta(4,5)=0 \\
\delta(1,4)=3 & \delta(3,2)=0 & \delta(3,4)=1 & \delta(5,4)=1
\end{array}
$$

Figure 4: Discounted distance

A weak (strong) component of a network $g$ is a subnetwork $\left.g\right|_{C}$, where $C \subseteq N$, such that for any two players $i, j \in C, \delta(i, j ; g)<\infty(\delta(i, j ; g)=0)$, and no set strictly containing $C$ meets this condition. We say that $g$ is weakly (strongly) connected if $g$ is the unique weak (strong) component of $g$. A weak (strong) component of a network is minimal if for all $i, j$ s.t. $g_{i j}=1$, the number of weak (strong) components of $g$ is smaller than the number of weak (strong) components of $g-i j$, where $g-i j$ is the network that results from replacing $g_{i j}=1$ by $g_{i j}=0$ in $g$. A network is minimally weakly (strongly) connected if it is weakly (strongly) connected and minimal. Note that when $0<\alpha<1$, weak (strong) connectedness means the existence of a path (oriented

\footnotetext{
${ }^{8}$ Note that $\delta(\cdot, \cdot ; g)$, though not a metric, satisfies the triangle inequality, i.e. $\delta(i, k ; g) \leq \delta(i, j ; g)+$ $\delta(j, k ; g)$.
} 
path) between any two players. When $\alpha=0$ (one-way flow) or $\alpha=1$ (two-way flow) the following is obtained:

$$
\delta(i, j ; g)<\infty \Leftrightarrow \delta(i, j ; g)=0
$$

therefore in both cases weak component and strong component are equivalent notions, and consequently those of (minimally or not) weakly connected network and strongly connected network are also equivalent. In fact, both variants of these notions are equivalent and coincide with what in the context of one-way and two-way flow models is called a component, connectedness and minimal connectedness in Bala and Goyal (2000a).

The set of players with whom $i$ supports a link is denoted by $N^{d}(i ; g)$, its cardinality by $\mu_{i}^{d}(g)$, and the set of players at a finite discounted distance from $i$ by

$$
N(i ; g):=\{j \in N: \delta(i, j ; g)<\infty\} .
$$

Thus each node $i$ receives some information from all nodes at a finite discounted distance from it. Let $v_{i j}>0$ be the payoff that player $i$ derives from $j$ when $\delta(i, j ; g)=0$, and $c_{i j}>0$ the cost for player $i$ of initiating a link with $j$. As $v_{i i}$ and $c_{i i}$ play no role in the strategic analysis, it is assumed that $v_{i i}=c_{i i}=0$. Thus, the payoff of player $i$ in $g$ is

$$
\Pi_{i}(g)=\sum_{j \in N(i ; g)} v_{i j} \alpha^{\delta(i, j ; g)}-\sum_{j \in N^{d}(i ; g)} c_{i j} .
$$

In what follows costs and benefits are assumed to be homogeneous across players (i.e. $v_{i j}=v$ and $c_{i j}=c$, for all $i \neq j$ ). It is also assumed that $v>c$, so that connecting with new nodes (i.e. those at discounted distance $\infty$ ) is always profitable. Without loss of generality it is assumed that $v=1$. In short, the following is assumed:

$$
\Pi_{i}(g)=\sum_{j \in N(i ; g)} \alpha^{\delta(i, j ; g)}-c \mu_{i}^{d}(g) \quad(c<1)
$$

The first term on the right-hand side of (1) represents the information that reaches player $i$ through network $g$ and the second its cost. Denoting them by

$$
I_{i}(g):=\sum_{j \in N(i ; g)} \alpha^{\delta(i, j ; g)}, \quad C_{i}(g):=c \mu_{i}^{d}(g),
$$

(1) can be rewritten as

$$
\Pi_{i}(g)=I_{i}(g)-C_{i}(g) \quad(c<1),
$$

that is: Payoff = Information - Cost.

One network is said to be more efficient than another when the aggregate payoff is greater. A network is efficient if it maximizes the aggregate payoff. 


\section{Stability}

Following Bala and Goyal (2000a), we consider two notions of stability. Let $g_{-i}$ denote the network where all links supported by $i$ in $g$ are deleted, and $\left(g_{-i}, g_{i}^{\prime}\right)$ the strategy profile and network that results from replacing $g_{i}$ by $g_{i}^{\prime}$ in $g$.

Definition 2 A Nash network is a network $g$ that is stable in the following sense: for all $i \in N$ :

$$
\Pi_{i}(g) \geq \Pi_{i}\left(g_{-i}, g_{i}^{\prime}\right) \quad \text { for all } g_{i}^{\prime} \in G_{i}
$$

When (2) holds, $g_{i}$ is said to be a best response of $i$ to $g_{-i}$. The stability notion can be refined in the strict sense:

Definition 3 A strict Nash network is a Nash network $g$ that is stable in the following stronger sense: for all $i \in N$ :

$$
\Pi_{i}(g)>\Pi_{i}\left(g_{-i}, g_{i}^{\prime}\right) \quad \text { for all } g_{i}^{\prime} \in G_{i}\left(g_{i}^{\prime} \neq g_{i}\right) .
$$

Thus, in a strict Nash network a unilateral change of strategy means a loss for the player who makes it. We first focus our attention on the strict stability, only when this issue is solved do we address the question of non-strict stability.

As a term of reference we first establish the range of values for the parameters within which the oriented wheel, the center-sponsored star and the oriented line are strict Nash networks. An oriented wheel, a wheel for short, ${ }^{9}$ is a network $g$ s.t. for a certain permutation of $N, i_{1}, i_{2}, . ., i_{n}$, we have $g_{i_{k} i_{k+l}}=1(k=1, . ., n-1)$, and $g_{n 1}=1$, and no other links exist. A center-sponsored star, a star for short, consists of a node (the center) that supports a link with each of the others and no other links exist. An oriented line, a line for short, is a network $g$ s.t. for a certain permutation of $N$, $i_{1}, i_{2}, . ., i_{n}$, we have $g_{i_{k} i_{k+l}}=1(k=1, . ., n-1)$, and no other links exist.

Proposition 1 In a society with $n$ players where payoffs are given by (1):

(i) The oriented wheel is strict Nash if and only if $c<n-1-\alpha-\alpha^{2}-\ldots-\alpha^{n-1}$.

(ii) The center-sponsored star is strict Nash if and only if $c>n-1-(n-1) \alpha$.

(iii) The oriented line is strict Nash if and only if $c>n-1-\alpha-\alpha^{2}-\ldots-\alpha^{n-1}$.

Proof. (i) In an oriented wheel all players receive all the information in the network without friction paying a single link each, i.e. a payoff of $n-1-c$. Thus the only possible best response of a player is to delete her/his single link, which would mean a payoff of $\alpha+\alpha^{2}+\ldots+\alpha^{n-1}$ for the player doing so. Therefore, the oriented wheel is

\footnotetext{
${ }^{9}$ In the literature the adjective "oriented" is often omitted, so an oriented wheel is usually called just a wheel. But given the importance of its oriented nature we choose to use the term at least in the statement of the results, omitting it sometimes in the text for the sake of brevity. The same applies for oriented lines and trees as defined bellow.
} 
strictly stable if $n-1-c>\alpha+\alpha^{2}+\ldots+\alpha^{n-1}$, which yields the necessary and sufficient condition.

(ii) In a center-sponsored star the center has no best response given that $c<1$ and consequently deleting a link would mean a loss. The payoff of each spoke agent is $(n-1) \alpha$, while initiating the most rewarding link, i.e. with the center, would mean $n-1-c$. Thus, the center-sponsored star is a strict Nash network if $(n-1) \alpha>n-1-c$, which yields the necessary and sufficient condition.

(iii) In an oriented line, given that $c<1$, deleting a link would mean a loss. The less demanding best response would be that of the player supporting no link and would consist of initiating a link with the player that receives all, i.e. $n-1$, without friction. The condition for this not to be a best response is the exact reverse of the one obtained for the oriented wheel. Observe that the only difference between the oriented wheel and the oriented line is whether or not a link exists.

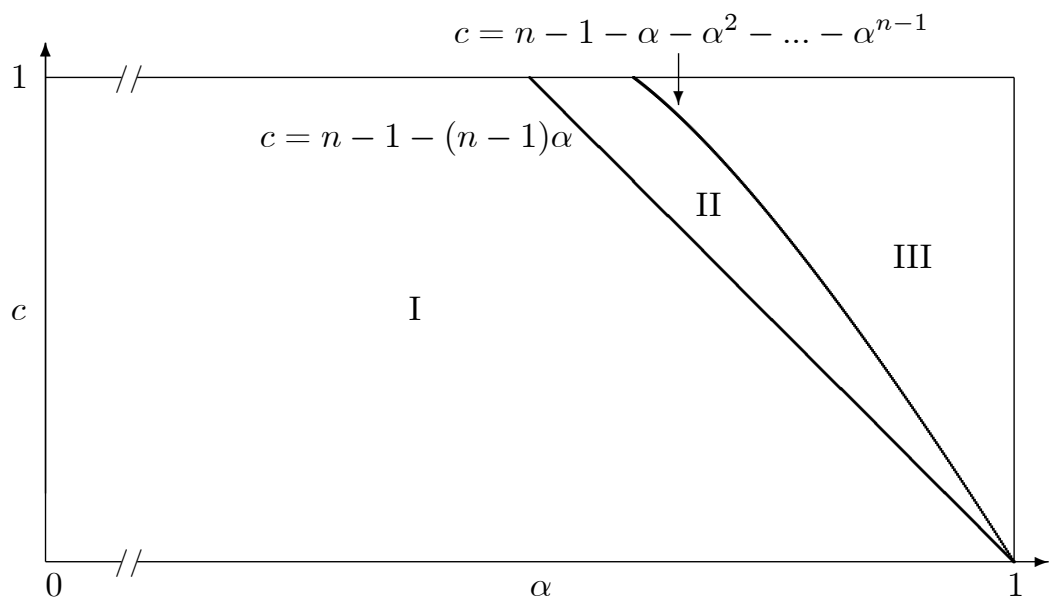

Figure 5: Ranges of strict stability for $n \geq 3$

Figure 5 shows the regions where each of these three architectures is strictly stable. The wheel is strict Nash below the polynomial function $c=n-1-\alpha-\alpha^{2}-\ldots-\alpha^{n-1}$ (i.e. in regions I and II), the center-sponsored star above the straight line $c=(n-$ $1)-(n-1) \alpha$ (regions II and III), so that both are strict Nash between these two lines (region II). The line becomes strictly stable just where the wheel ceases to be so (region III). As the number of players grows, the range of values of the parameters for which the wheel is strictly stable widens while those for the line and the star shrink.

Note that Figure 2 represents the particular case for $n=3$ of Figure 5 . For $n=3$ there are no more strict stable architectures but this is not so for $n>3$. In particular, many more oriented trees other than the center-sponsored star and the oriented line appear, each of them strictly stable for a certain range of the parameters as we presently show. A root-oriented tree is a network where there is a single node $i_{0}$, called the root, such that for any other node $j$ there is a unique path from $j$ to $i_{0}$, and that path is 
$i_{0}$-oriented. Observe that in a root-oriented tree all the information in the tree reaches the root without friction (at the cost of as many links as the root supports), while all other nodes receive some of the information in the network with some loss. Note also that on going further down the tree away from the root by any path the amount of information that reaches a node diminishes. In particular, a terminal node pays no link but receives less information than its predecessors closer to the root. The following notation proves useful. Let $g$ be an $i_{0}$-oriented tree, $i$ a node and assume that the length of the path connecting $i_{0}$ and $i$ is $r$. Notice that then the maximal discounted distance from any node in the tree to $i$ is $r$. Then there is a partition of $N \backslash i$ into $r$ disjoint sets $N_{i 0}, N_{i 1}, N_{i 2}, . ., N_{i r}$, where

$$
N_{i s}:=\{j \in N \backslash i: \delta(i, j ; g)=s\} .
$$

Thus, denoting the cardinality of $N_{i s}$ by $n_{i s}$, the payoff of a node $i$ in a root-oriented tree is given by

$$
\Pi_{i}(g)=n_{i 0}+n_{i 1} \alpha+n_{i 2} \alpha^{2}+\ldots+n_{i r} \alpha^{r}-c \mu_{i}^{d}(g) .
$$

Note that for a terminal node in the tree, as $n_{i 0}=0$ and $\mu_{i}^{d}(g)=0$, the following is obtained

$$
\Pi_{i}(g)=I_{i}(g)=n_{i 1} \alpha+n_{i 2} \alpha^{2}+\ldots+n_{i r} \alpha^{r} .
$$

Then the following result emerges concerning the stability of trees.

Proposition 2 A root-oriented tree $g$ is a strict Nash network if and only if: $\alpha=1$ and $g$ is a center-sponsored star; or $\alpha<1$ and $I_{i}(g)>n-1-c$ for all terminal nodes.

Proof. Let $g$ be a root-oriented tree. As $c<1$ no deletion can be a part of a best response. If $\alpha=1$ and there is a path, $i_{2}, i_{1}, i_{0}$, of length 2 from a node $i_{2}$ to the root $i_{0}$, then replacing its link with $i_{1}$ by a link with $i_{2}$ is a best response of $i_{0}$ (same payoff) and $g$ is not strict Nash. Therefore if $\alpha=1$ the only root-oriented tree that is strict Nash is the center-sponsored star. If $\alpha<1$ the root has no best response, and the only possible best response of a player other than the root is to initiate a link with the root. For this not to be a best response, the current payoff must be greater than what $\mathrm{s}$ /he would receive by initiating that link. That is, using (4), for all nodes other than the root must be

$$
n_{i 0}+n_{i 1} \alpha+n_{i 2} \alpha^{2}+\ldots+n_{i r} \alpha^{r}-c \mu_{i}^{d}(g)>n-1-c-c \mu_{i}^{d}(g),
$$

or equivalently

$$
n_{i 0}+n_{i 1} \alpha+n_{i 2} \alpha^{2}+\ldots+n_{i r} \alpha^{r}>n-1-c .
$$

That is, $I_{i}(g)>n-1-c$. The same condition is necessary for all players other than the root, but it is terminal players whose payoffs would improve most if they initiate a link with the root (they receive less information than their predecessors). In other 
words it is for terminal players that this condition is most demanding. Thus, $g$ is a strict Nash network if and only if the following condition holds for all terminal node $i$ :

$$
I_{i}(g)=n_{i 1} \alpha+n_{i 2} \alpha^{2}+\ldots+n_{i r} \alpha^{r}>n-1-c .
$$

Note that when $\alpha=0$ this condition cannot be met, i.e. no root-oriented tree is strict Nash in this case.

Observe that because the oriented line and the center-sponsored star are rootoriented trees parts (ii) and (iii) of Proposition 1 are now corollaries of Proposition 2. Nevertheless, the range of strict stability which has been established for each of the three architectures in Proposition 1 allows a clearer idea of the ranges of the new ones yet to be established. In particular, Figure 5 region II gives upper and lower bounds for the region where any other root-oriented tree can be strictly stable. Note that the bound for the cost $c$ in terms of $\alpha$ for any root-oriented tree is of the form

$$
c>n-1-\left(n_{i 1} \alpha+n_{i 2} \alpha^{2}+\ldots+n_{i r} \alpha^{r}\right),
$$

for a terminal node, but observe that whatever the root-oriented tree is, for all $\alpha$ $(0<\alpha<1)$ :

$$
\alpha+\alpha^{2}+\ldots+\alpha^{n-1}<n_{i 1} \alpha+n_{i 2} \alpha^{2}+\ldots+n_{i r} \alpha^{r}<(n-1) \alpha .
$$

For this, observe that the three terms that appear in (7) are polynomials whose coefficients are positive integers that add up to $n-1$, given that each of them is the result of adding each of $n-1$ nodes times its discounted distance to the terminal node of a root-oriented tree. ${ }^{10}$ Therefore the concave curve above which a root-oriented tree is strictly stable is between the straight line corresponding to the center-sponsored star and the curve corresponding to the oriented line, i.e. it is within region II in Figure 5.

In sum, at this stage it results that oriented wheels and root-oriented trees are strict Nash networks for certain ranges of values of the parameters. As we show below, there is a still more complex architecture that can be stable, in fact an architecture that incorporates all the preceding ones as particular cases, which we call "wheel of trees" or "hybrid vortex". A wheel of trees or hybrid vortex is a network which consists of a set of nodes $i_{1}, i_{2}, . ., i_{k}(k \geq 1)$ arranged as an oriented wheel if $k>1$ (i.e., $g_{i_{l} i_{l-1}}=1$ for $l=2, . ., k, g_{i_{1} i_{k}}=1$ and no more links between them exist), plus a "tributary" $i_{l^{-}}$ rooted root-oriented tree $t_{i_{l}}$ for some of them (see Figure 6). That is, each $i_{l}$ belonging to the central wheel can be the root of a root-oriented tree $t_{i_{l}}$. Let $w_{i_{l}}$ denote the "weight" (i.e. the number of nodes) of tree $t_{i_{l}}$, with $w_{i_{l}}=1$ if no tree hangs from $i_{l}$. In a minimally weakly connected network with such an architecture all nodes in the central wheel (and they alone in the network if $\alpha<1$ ) receive all the information from

\footnotetext{
${ }^{10}$ The particular structure of such polynomials makes it easy to make comparisons within the interval $0<\alpha<1$, where $\alpha>\alpha^{2}>\alpha^{3}$. . Note that given one such polynomial, any "transfer of weight" from a coefficient of, say $\alpha^{k}$, to that of smaller power $\alpha^{l}(l<k)$ increases its value.
} 
all the nodes through the tributary trees without loss. ${ }^{11}$ Observe also that this structure includes the oriented wheel (when no tree hangs from any node in the central wheel or, equivalently, if $k=n$ ), and the oriented tree (when the central wheel collapses into a single node or, equivalently, if $k=1$ ). In terms of this notation and terminology the following result emerges.

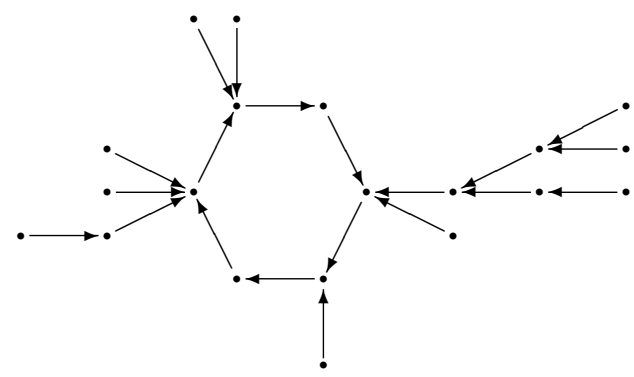

Figure 6: A wheel of trees

Proposition $3 A$ wheel of trees $g$ consisting of a wheel with nodes $i_{1}, i_{2}, . ., i_{k}$ and tributary trees $t_{i_{l}}(l=1,2, . ., k)$ is strict Nash if and only if

$$
n-1-\left(I_{j}\left(t_{i_{l}}\right)+\alpha^{\delta\left(j, i_{l} ; t_{i_{l}}\right)} \sum_{r \neq l} w_{i_{r}}\right)<c<n-\left(w_{p\left(i_{1}\right)}+w_{p\left(i_{2}\right)} \alpha+\ldots+w_{p\left(i_{k}\right)} \alpha^{k-1}\right),
$$

for all $l=1,2, . ., k$, all terminal node $j \in t_{i_{l}}$, and all cyclic permutation $p$ of the finite sequence $i_{1}, i_{2}, . ., i_{k}$.

Proof. In a wheel of trees there are only two possible types of best response: that of a player in the wheel deleting her/his wheel-link, and that of a player not in the wheel initiating a link with any player in the wheel. For the first action not to be a best response the condition is similar to the one obtained for the wheel in Proposition 1 , but now each of the nodes in the wheel has a "weight" other than 1 if a tree hangs from it. In order for a player, say $i_{1}$, in the wheel to lose by deleting her/his wheel-link the following inequality must hold

$$
w_{i_{1}}-1+w_{i_{2}} \alpha+\ldots+w_{i_{k}} \alpha^{k-1}<n-1-c
$$

or equivalently

$$
c<n-\left(w_{i_{1}}+w_{i_{2}} \alpha+\ldots+w_{i_{k}} \alpha^{k-1}\right) .
$$

\footnotetext{
${ }^{11}$ We have been compelled to invent a name for these new structures. "Wheel of trees" seems a rather descriptive name, while "vortex" was suggested by their similarity to a spinning flow of fluid, and "hybrid" because of their mixed character. We have chosen to use the first of these names in the rest of the paper.
} 
Thus, this condition must hold for all the nodes in the wheel (i.e. replacing $i_{1}$ by any other $i_{r}$ in the role of "deleter"). In other words, it must hold for any cyclic permutation of $i_{1}, i_{2}, . ., i_{k}$ which yields the upper bound for $c$.

As to the players not in the wheel, since each of them is in one of the tributary trees a condition similar to the one obtained in Proposition 2 for the trees emerges, but now the root, say $i_{k}$, accumulates a weight of $w_{i_{1}}+w_{i_{2}}+\ldots+w_{i_{k-1}}$. Again the most demanding condition is for some of the terminal nodes. Namely, the following condition is needed: for any node $i_{l}$ in the wheel and any terminal node $j$ in that tree $t_{i_{l}}$ whose information received from the tributary tree it belongs to is $I_{j}\left(t_{i_{l}}\right)$

$$
I_{j}\left(t_{i_{l}}\right)+\alpha^{\delta\left(j, i_{l} ; t_{i_{l}}\right)} \sum_{r \neq l} w_{i_{r}}>n-1-c,
$$

must hold, or equivalently

$$
c>n-1-\left(I_{i}\left(t_{i_{l}}\right)+\alpha^{\delta\left(j, i_{l} ; t_{i}\right)} \sum_{r \neq l} w_{i_{r}}\right),
$$

which yields the lower bound for $c$.

Remarks: (i) Note that a wheel of trees shares to some extent the natures of both a tree and a wheel. Thus we obtain for $c$ both a lower bound (as for oriented trees) and an upper bound (as for the oriented wheel).

(ii) In fact, Proposition 3 contains Propositions 1 and 2. To see this note that when the central wheel collapses into a node (i.e. $k=1$ ), only the lower bound for $c$ remains in (8) and becomes exactly that for terminal nodes in Proposition 2. When no tree hangs from any node in the central wheel (i.e. $k=n$ ) only the upper bound for $c$ remains in (8) and, as all weights are 1, this bound becomes exactly that in (i) in Proposition 1.

(iii) At first sight the bounds for $c$ between which a wheel of trees remains strict Nash may seem somewhat obscure. However, it is easy to see exploiting their polynomial form that both bounds lie within region II in Figure 5 (between the straight line $c=$ $n-1-(n-1) \alpha$ and the polynomial function $\left.c=n-1-\alpha-\alpha^{2}-\ldots-\alpha^{n-1}\right)$ where the wheel and the center-sponsored stars are strictly stable. In other words, the region where a wheel of trees is strictly stable is contained within the region where both the wheel and the center-sponsored star are strictly stable.

(iv) It may well be the case that for a certain wheel of trees the lower bound for $c$ is greater than the upper bound for any $\alpha(0<\alpha<1)$, in which case it is never a strict Nash network. For instance, in the three-player case the wheel of trees where one player supports a link with each of the other two and only one of them supports a link with it is not a strict Nash network whatever the values of the parameters.

(v) For the interval defined by (8), within which a wheel of trees is strictly stable, not to be empty a delicate trade-off balance is required. Some examples help to understand this better. 
Example 1: Consider the $m(n+1)$-node wheel of trees consisting of $m$ nodes arranged as an oriented wheel and each of them supporting links with $n$ different nodes. Given the symmetry of the network, conditions (8) yield the following conditions on the parameters for such an architecture to be a strict Nash network:

$$
(m n+m-1)(1-\alpha)<c<m n+m-(n+1)\left(1+\alpha+\alpha^{2}+\ldots+\alpha^{m-1}\right) .
$$

Which, for instance, if $m=6, n=3$ and $\alpha=0.97$, gives that this architecture is strict Nash for any cost in the interval ${ }^{12} 0.69<c<1$.

Example 2: Consider the $(m+n)$-node wheel of trees consisting of $m$ nodes arranged as an oriented wheel and only one of them supporting links with $n$ nodes. In this case (8) yield the following conditions on the parameters:

$$
(m n+m-1)(1-\alpha)<c<m-\left(1+\alpha+\alpha^{2}+\ldots+\alpha^{m-1}\right) .
$$

By comparing the two curves within the interval $0 \leq \alpha \leq 1$, it is easy to conclude that there is room between them only if $m^{3}-3 m>2 n-2$. Thus, for instance, if $m=6$, then it must be that $n<10$ for the above interval to be nonempty. As a numerical example, if $m=n=6$ and $\alpha=0.95$, this architecture is strictly stable within the interval of cost $0.55<c<0.70184$.

Proposition 3 gives the range of parameters for which a wheel of trees is strict Nash. We now show that this is the only possible architecture of a strict Nash asymmetric flow network. The following straightforward lemma is most useful in characterizing strict Nash networks.

Lemma 1 If $g$ is a strict Nash network then no three nodes $i, j, k$ exist such that $g_{i k}=g_{j k}=1$.

Proof. Assume $g_{i k}=g_{j k}=1$ with $i \neq j$. Then all the information that reaches $k$ also reaches $j$ without decay through link $\overleftarrow{j k}$, and player $i$ can replace link $\overleftarrow{i k}$ by link $\overleftarrow{i j}$ without loss. Therefore $g$ is not a strict Nash network.

Theorem 1 In the asymmetric flow model a network is strict Nash if and only if it is a weakly connected wheel of trees that satisfies (8).

Proof. As sufficiency was established in Proposition 3, it only remains to show necessity. Let $g$ be a strict Nash network. Assume that $g$ contains a sequence of players $i_{1}, i_{2}, . ., i_{k}$, arranged as a wheel (i.e. $\bar{g}_{i_{l} i_{l-1}}=1$ for $l=2, . ., k, \bar{g}_{i_{1} i_{k}}=1$ ). From Lemma 1 it follows that they must form an oriented wheel. If it contains all players we are done. Otherwise, as $g$ is strict Nash and $c<1$, any other node must be connected to the wheel and, again by Lemma 1 , this connection must be by an $i_{l}$-oriented path

\footnotetext{
${ }^{12}$ In fact, the upper bound given by (8) is 1.729 , but the model assumes $c<1$.
} 
with an $i_{l}$ in the wheel. Moreover, for each player not in the wheel the oriented path connecting it to the wheel must be unique, otherwise Lemma 1 would be contradicted. Thus the architecture implied is that of a wheel of trees. Now assume that $g$ does not contain a sequence of players arranged as a wheel. Let Prd (for "predecessor") be the binary relation defined by

$$
i \operatorname{Prd} j \Leftrightarrow i \stackrel{g}{\longleftarrow} j \text { or } i=j \text {. }
$$

This relation is reflexive, antisymmetric (note that $i \stackrel{g}{\longleftarrow} j$ and $j \stackrel{g}{\longleftarrow} i$ would imply the existence of a wheel) and transitive. As $g$ is strict Nash, there must be a path connecting any two players $i$ and $j$. From Lemma 1, necessarily either $i \stackrel{g}{\longleftarrow} j$ or $j \stackrel{g}{\longleftarrow} i$ or there is a third player $k$ s.t. $i \stackrel{g}{\longrightarrow} k \stackrel{g}{\longleftarrow} j$. In other words, for any two players there is a common predecessor. But then there must be a unique predecessor of all players, i.e. a player $i_{0}$ such that for every other player $j$ there is a path $i_{0} \stackrel{g}{\longleftarrow} j$. And from Lemma 1 that path must be unique. Thus $g$ is an $i_{0}$-oriented tree.

Conclusion 1 Summarizing the study of strict Nash asymmetric flow networks the following picture emerges as one moves from south-west to north-east in the rectangle containing all pairs of values of the two parameters of the model (or from $\alpha=0$ to $\alpha=1$ along any horizontal straight line $c=$ constant). First only the oriented wheel is strict Nash (region I in Figure 5), then the center-sponsored star becomes also strict Nash. Then, as one goes further right, other oriented trees also become strict Nash until the last tree, i.e. the oriented line, also becomes strict Nash. In this range of values for the parameters (region II) where oriented wheels and trees coexist as strict Nash some wheels of trees also appear as strict Nash but, unlike the oriented trees that remain strict Nash as a increases, these hybrid creatures remain strict Nash only ephemerally for a narrow subset ${ }^{13}$ of values within region II. Finally, as soon as the last tree (the oriented line) joins the set of strict Nash networks the wheel abandons it. All oriented trees remain strict Nash in region III, but when $\alpha=1$ a brusque discontinuity occurs and only the center-sponsored star survives as strict Nash.

Remark: It is worth noting the different images of the two limiting cases, $\alpha=0$ and $\alpha=1$, from the point of view provided by the asymmetric flow model. The case $\alpha=0$ appears as just the case of maximal asymmetry and the conclusions about strict Nash networks for this case (i.e. the one-wayflow model) remain unchanged for a wide range of values of the parameters. In contrast, when asymmetry disappears for $\alpha=1 \mathrm{a}$ brusque discontinuity occurs: for values of $\alpha$ slightly smaller than 1 all oriented trees are strict Nash, but only the center-sponsored star remains strict Nash for $\alpha=1$.

We now address the structure of non-strict Nash networks. As $c<1$, the obvious first conclusion is that Nash networks are weakly connected. The following weak version of Lemma 1 is rich in implications about their architecture.

\footnotetext{
${ }^{13}$ Note that this "narrowness" concerns particularly to $\alpha$, but for each $\alpha$ within that narrow interval it may well be the case that a wheel of trees remains strictly stable for a relatively wide range of cost. See, for instance, Example 1.
} 
Lemma 2 If $g$ is a Nash network and $g_{i k}=g_{j k}=1$ for three nodes $i, j, k$, then link $\overleftarrow{i k}$ is part of an oriented wheel and link $\overleftarrow{j k}$ is part of another oriented wheel.

Proof. Let $g$ be a Nash network and assume $g_{i k}=g_{j k}=1$. As all the information that reaches $k$ also reaches $j$ without decay through link $\overleftarrow{j k}$, player $i$ can replace link $\overleftarrow{i k}$ by link $\overleftarrow{i j}$ without loss. But also without gain given that $g$ is a Nash network. This implies that $k$ receives the unit of $j$ 's worth without decay in $g$. Thus, there is a path $j \stackrel{g}{\longrightarrow} k$ that with $\overleftarrow{j k}$ completes a wheel. The same conclusion is obtained similarly for link $\overleftarrow{i k}$

The following lemma provides information about the structure of Nash networks.

Lemma 3 Let $g$ be a Nash network. If $g$ contains two oriented wheels, $W$ and $W^{\prime}$, there is a sequence of oriented wheels $W_{1}, . ., W_{k}$ s.t. $W \cap W_{1} \neq \varnothing, W^{\prime} \cap W_{k} \neq \varnothing$, and $W_{l} \cap W_{l+1} \neq \varnothing(l=1, . ., k-1)$.

Proof. If $W \cap W^{\prime} \neq \varnothing$ we are done. Assume $W \cap W^{\prime}=\varnothing$. Assume first $0<\alpha<1$. As $g$ is weakly connected, there must exist a node $i \in W$ and a node $i^{\prime} \in W^{\prime}$ connected by a path $i_{0}, i_{1}, . ., i_{k}$, s.t. $i=i_{0}, i^{\prime}=i_{k}$, and for all $l=0, . ., k-1, \bar{g}_{i_{l} i_{l+1}}=1$. Assume first that this path is oriented, say, without loss of generality, $i^{\prime}$-oriented, then $i=i_{0}$ is linked by a node in $W$ and by $i_{1}$. Then, by Lemma 2 , link $i_{1} i_{0}$ is part of an oriented wheel $W_{1}$. By reiterating this step, applying Lemma 2 repeatedly, the desired sequence of wheels is proved to exist. If the path connecting $i$ and $i^{\prime}$ is not oriented, then at a node $i_{l}$ in the path either $g_{i_{l-1} i_{l}}=g_{i_{l+1} i_{l}}=1$ (case 1 ), or $g_{i_{l} i_{l-1}}=g_{i_{l} i_{l+1}}=1$ (case 2 ). In the first case Lemma 2 applies to both links, and can be repeatedly applied in either direction as far as the links have the same orientation as the preceding ones until either $W$ or $W^{\prime}$ is reached or an inverted link is met, but then the second case arises. In this case the reversed link has its tail closer to a wheel than its tip, so that the subsequent links either all have this orientation until the wheel is reached and Lemma 2 can be applied as in the oriented case, or at some point case 1 emerges again. Thus, whatever the path connecting $i$ and $i^{\prime}$, each of its links belongs to an oriented wheel. Now assume $\alpha=0$. In this case, weak connectedness is equivalent to strong connectedness, so that there is an oriented path connecting any two players. This is the first case considered when $0<\alpha<1$, and by an identical argument the same conclusion is obtained. As to the case $\alpha=1$, the lemma holds trivially given that no wheel can be part of a Nash network in this case.

Thus, a Nash network either contains no oriented wheel or they are all interconnected. A maze of oriented wheels within a network is referred to as a subnetwork consisting of a set of oriented wheels such that for any two wheels $W$ and $W^{\prime}$ in this set there is a sequence of oriented wheels in the set s.t. the first is $W$, the last is $W^{\prime}$, and any two consecutive wheels in the sequence share at least one node. In order to simplify the statement of the next result the degenerate case of a single node is included as a maze of oriented wheels. This gives the following result. 
Theorem 2 In the strictly asymmetric flow model the only possible architecture of a Nash network is that of a weakly connected network with at most one strong component (consisting of a maze of wheels which is minimally strongly connected) plus a number of root-oriented trees whose roots belong to the maze.

Proof. First assume that $g$ contains an oriented wheel. Therefore, from Lemma 3 all oriented wheels are interconnected and form a maze of wheels. If the maze contains all nodes we are done. Otherwise, by reasoning exactly as in Theorem 1, the conclusion is that any other player lies in a root-oriented tree whose root belongs to the maze. If the network does not contain any oriented wheels, in view of Lemma 2 no three nodes $i, j, k$ exist such that $g_{i k}=g_{j k}=1$. But this was the conclusion of Lemma 1 for any strict Nash network. So by reasoning exactly as in Theorem 1, it can be concluded that $g$ is a root-oriented tree.

Remarks: (i) Theorem 2 establishes the only possible architecture of a Nash network in the strictly asymmetric flow model, ${ }^{14}$ but not every network with this architecture is a Nash network. As was the case for strict Nash networks, for this to be true some conditions involving the parameters must hold. First, every terminal node of the trees sets a lower bound for $c$ similar to the one in (8), but now with non-strict inequality. Second, every link in the maze sets an upper bound for $c$, but now the variety of mazes makes it impossible to formulate them as precisely as for a strict Nash network beyond the following general condition: for every node $i$ in the maze, and every $j \in N^{d}(i ; g)$,

$$
\Pi_{i}(g-i j)=I_{i}(g-i j)-c\left(\mu_{i}^{d}(g)-1\right) \leq n-1-c \mu_{i}^{d}(g),
$$

or equivalently, $c \leq n-1-I_{i}(g-i j)$.

(ii) Observe that a strict Nash network is a Nash network whose "maze" consists of at most one single oriented wheel.

(iii) In a Nash network the nodes in the maze receive all the information from the network without decay (i.e. $I_{i}(g)=n-1$ for all $i$ in the maze), while in the periphery of tributary oriented trees each node receives without decay only the information from the nodes in the lower part of the tree that it belongs to, while information from all other nodes is received with decay.

(iv) When $\alpha=0$ the weak and strong variants of minimal connectedness are equivalent to minimal connectedness, which eliminates the trees. Thus the conclusion of Theorem 2 can be rewritten like this for this particular case:

Corollary 1 When $\alpha=0$ (i.e. in the one-way flow model) a Nash network is a maze of wheels minimally connected.

\footnotetext{
${ }^{14}$ For instance, for the tree-player case discussed in the introduction the only non-strict Nash network (for $c \leq 1-\alpha$ ) consists of four links, where one player links with the other two and is linked by both, but note that this is a maze consisting of two two-player wheels.
} 


\section{Efficiency}

A comparative study of the relative efficiency of the oriented wheel, the center-sponsored star and the oriented line such as the one in the introduction yields the following results. In an $n$-player society the aggregate payoff of the oriented wheel is

$$
W=\sum_{\text {wheel }} u_{i}=n(n-1-c),
$$

the aggregate payoff of the center-sponsored star is

$$
S=\sum_{\text {star }} u_{i}=(n-1)(n-1) \alpha+(n-1)(1-c),
$$

and the aggregate payoff of the oriented line is

$$
L=\sum_{\text {star }} u_{i}=(n-1) \alpha+(n-2) \alpha^{2}+\ldots+2 \alpha^{n-2}+\alpha^{n-1}+\frac{n(n-1)}{2}-(n-1) c,
$$

which for $\alpha \neq 1$ can be rewritten as

$$
L=\frac{n(n-1)}{2}+\frac{(n-1) \alpha}{1-\alpha}-\frac{\left(1-\alpha^{n-1}\right) \alpha^{2}}{(1-\alpha)^{2}}-(n-1) c .
$$

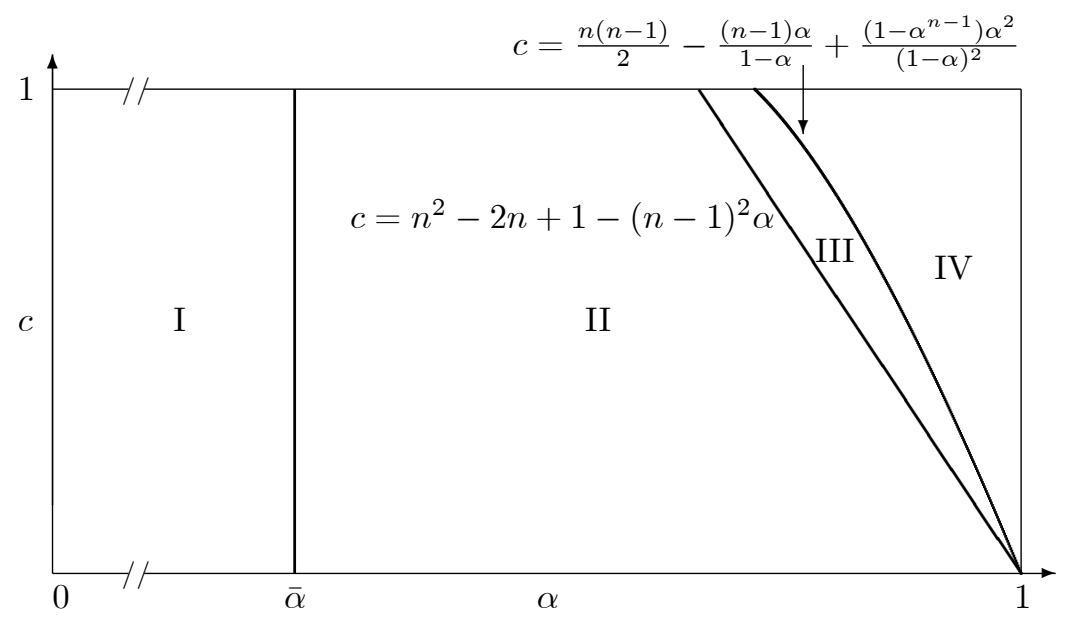

Figure 7: Ranges of efficiency for $n>3$

Comparing these figures, $W, S$ and $L$, the situation described on Figure 7 emerges. The rectangle of possible values of the parameters is divided into four regions. In region I, when $\alpha<\bar{\alpha}$, where $\bar{\alpha}$ is the solution of $\frac{(n-1)(n-2)-2(n-1)^{2} \alpha}{2}+\frac{(n-1) \alpha}{1-\alpha}-\frac{\left(1-\alpha^{n-1}\right) \alpha^{2}}{(1-\alpha)^{2}}=0,{ }^{15}$ we have

$$
W>L>S \text {. }
$$

\footnotetext{
${ }^{15}$ Equating $L$ and $S$ yields this equation, which has a unique solution in the interval $(0,1)$.
} 
In region II, when $\alpha>\bar{\alpha}$ and $c<n^{2}-2 n+1-(n-1)^{2} \alpha$ :

$$
W>S>L \text {. }
$$

In region III, when $n^{2}-2 n+1-(n-1)^{2} \alpha<c<\frac{n(n-1)}{2}-\frac{(n-1) \alpha}{1-\alpha}+\frac{\left(1-\alpha^{n-1}\right) \alpha^{2}}{(1-\alpha)^{2}}$ :

$$
S>W>L \text {. }
$$

In region IV, when $c>\frac{n(n-1)}{2}-\frac{(n-1) \alpha}{1-\alpha}+\frac{\left(1-\alpha^{n-1}\right) \alpha^{2}}{(1-\alpha)^{2}}$ :

$$
S>L>W \text {. }
$$

Thus, the oriented line is always beaten by the wheel or the star but, unlike in the three-player case discussed in the introduction, it may be that none of these structures is efficient. For instance, for $n=5$ and a certain range of values, the efficient network is an oriented tree of length 2 where the root supports only one link. A comparison with the two benchmark models is pertinent here. In the two-way flow model all Nash networks, strict or not, are efficient, while in the one-way flow model not all Nash networks are efficient, but the strict Nash networks (i.e. the oriented wheels) are. In contrast, as seen in the three-player case and as is the case in general, in the asymmetric flow model Nash and strict Nash networks may not be efficient and efficient networks may not be stable. In other words, efficiency and stability do not in general go hand in hand in the asymmetric flow model.

\section{Dynamics}

We now consider Bala and Goyal's (2000) dynamic model in this setting. Namely, starting from any initial network $g$, in each period each player $i$ responds, independently from the others, with a positive probability with a best response to $g_{-i}$ (this includes any strategy that yields the same payoff to $i$ as the current one when no strategy can improve $i$ 's payoff), or randomizes across them when there are more than one. Otherwise, player $i$ exhibits inertia, i.e. keeps her/his links unchanged. In this way, a Markov chain on the state space of all networks is defined. Bala and Goyal (2000) proves that this dynamic model converges to the oriented wheel for the one-way flow model and to the center-sponsored star for the two-way flow model. They prove this by showing that starting from any network a finite sequence of best responses exists that leads to a strict Nash network. In the current setting, as seen above, depending on the parameters $\alpha$ and $c$ and on the number of players, beyond the region where the oriented wheel is the only one there is a rich family of strict Nash networks. Nevertheless, we are able to prove that generically, i.e. save for at most a subset of zero measure of the rectangle of feasible values of the parameters, this dynamic process converges to a strict Nash network. Namely, we have the following result.

Theorem 3 In the asymmetric flow model best response dynamics converge generically to a strict Nash network with probability 1. 
Proof. The cases $\alpha=0$ and $\alpha=1$ are covered by Bala and Goyal's (2000) results. Thus, it suffices to show that for $0<\alpha<1$, save for at most a subset of zero measure of the rectangle of feasible values of the parameters, starting from any network, a sequence of best responses exists where at each stage a single node plays a best response while the others exhibit inertia that ends up at a strict Nash network. The proof is given in three steps. First, we show that from any network a finite sequence of best responses exists which yields a network where at least one player receives all the information without loss (Lemma 4). We then prove that from any such network a sequence of best responses exists which yields a wheel of trees where all nodes except perhaps those in the central wheel play their unique best responses. Finally, we show that starting from any such network a sequence of best responses exists which yields a strict Nash network. Given the complexity of generating such sequences we provide an algorithmic proof of the second and third steps, proving that the second one necessarily terminates and the third one generically terminates and yields a strict Nash network.

The following lemma provides the first step.

Lemma 4 Let $g$ be any network, then either there is a player that receives all the information without loss (i.e. $I_{i}(g)=n-1$ for at least one $i$ ) or there is a finite sequence of best responses that yields a network where at least one player receives all the information without loss.

Proof. The cases $\alpha=0$ and $\alpha=1$ are covered by Bala and Goyal's (2000) results. Thus we assume $0<\alpha<1$. Assume that $g$ is a network where no player receives all the information without loss (i.e. for all $i, I_{i}(g)<n-1$ ). Then, in view of Theorem $1, g$ is not a strict Nash network, and consequently at least one player has a best response. Let one of these players play a best response while all other players maintain their current strategies. Since $c<1$ this player must be connected by a path with any other player in the resulting network $g^{\prime}$, and, as $\alpha>0, g^{\prime}$ must be weakly connected. Then choose a node $i_{0}$ among those that receive the largest amount of information in $g^{\prime}$. If $I_{i_{0}}\left(g^{\prime}\right)=n-1$ we are done. Otherwise, there is a node from which the discounted distance to $i_{0}$ is $>0$. Let $i$ be a node for which $\delta\left(i_{0}, i ; g^{\prime}\right)$ is maximal. Then choose a node $j$ among those s.t. $\delta\left(i, j ; g^{\prime}\right)=0$ and $\delta\left(i_{0}, j ; g^{\prime}\right)=\delta\left(i_{0}, i ; g^{\prime}\right)$ for which the length of the shortest $i$-oriented path to $i$ is the largest (if there is no such a node $j \neq i$, then take $j=i$ ). Let $g^{\prime \prime}$ be the network that results from $j$ deleting all $j$ 's links and adding ${ }^{16}$ only one with $i_{0}, \overleftarrow{j i_{0}}$. Let us see that $I_{j}\left(g^{\prime \prime}\right)-I_{i_{0}}\left(g^{\prime}\right)>0$ and find a lower bound for this difference. Assume that $j$ supports a link $\overleftarrow{j j^{\prime}}$ in $g^{\prime}$, then either $\delta\left(i_{0}, j^{\prime} ; g^{\prime}\right)<\delta\left(i_{0}, i ; g^{\prime}\right)$, or $\delta\left(i_{0}, j^{\prime} ; g^{\prime}\right)=\delta\left(i_{0}, i ; g^{\prime}\right)$ and there is a shorter $i$-oriented path from $j^{\prime}$ to $i$. In both cases the deletion of $\overleftarrow{j j^{\prime}}$ cannot damage $i_{0}$ 's sight of $j^{\prime}$, nor damage $i_{0}$ 's sight of $j$ because as, in addition to all deletions, $j$ initiates a link with $i_{0}$,

\footnotetext{
${ }^{16}$ Note that necessarily $g_{j i_{0}}^{\prime}=0$, otherwise, given the choice of $j, I_{j}\left(g^{\prime}\right)>I_{i_{0}}\left(g^{\prime}\right)$, which contradicts the choice of $i_{0}$.
} 
we have $\delta\left(i_{0}, j ; g^{\prime \prime}\right)=1 \leq \delta\left(i_{0}, j ; g^{\prime}\right)$. So $j$, by a single link with $i_{0}$, receives in $g^{\prime \prime}$ all the information received by $i_{0}$ in $g^{\prime}$ minus that of node $j$ itself (i.e. $\alpha^{\delta\left(i_{0}, j ; g^{\prime}\right)}=\alpha^{\delta\left(i_{0}, i ; g^{\prime}\right)}$ ), plus that of node $i_{0}$ now without decay, that is:

$$
I_{j}\left(g^{\prime \prime}\right)=I_{i_{0}}\left(g^{\prime}\right)+1-\alpha^{\delta\left(i_{0}, i ; g^{\prime}\right)},
$$

and consequently as $j$ supports only one link in $g^{\prime \prime}$ and as $i_{0}$ supports at least one in $g^{\prime}$ (otherwise $i_{0}$ cannot be the node that receive the largest amount of information in $g^{\prime}$ ):

$$
\begin{gathered}
\Pi_{j}\left(g^{\prime \prime}\right)=I_{j}\left(g^{\prime \prime}\right)-1=I_{i_{0}}\left(g^{\prime}\right)-\alpha^{\delta\left(i_{0}, i ; g^{\prime}\right)}=\Pi_{i_{0}}\left(g^{\prime}\right)+C_{i_{0}}\left(g^{\prime}\right)-\alpha^{\delta\left(i_{0}, i ; g^{\prime}\right)} \\
\geq \Pi_{i_{0}}\left(g^{\prime}\right)+1-\alpha^{\delta\left(i_{0}, i ; g^{\prime}\right)} \geq \Pi_{i_{0}}\left(g^{\prime}\right)+1-\alpha .
\end{gathered}
$$

Thus $j$ has a response that means a payoff in $g^{\prime \prime}$ at least as large as that of $i_{0}$ in $g^{\prime}$ plus $1-\alpha>0$. This may or not be a best response, but it means that $j$ has a best response yielding a network $\bar{g}$ where $j$ 's payoff is at least

$$
\Pi_{j}(\bar{g}) \geq \Pi_{i_{0}}\left(g^{\prime}\right)+1-\alpha>\Pi_{i_{0}}\left(g^{\prime}\right) .
$$

Then, as an obvious upper bound for the possible payoff of a player in a network with $n$ nodes is $n-1$, only a finite number of repetitions of this argument (based on the assumption that no node receives all the information without loss) is possible. In other words, a finite number of repetitions of this argument necessarily yields a network where at least one node receives all the information without loss.

In view of Lemma 4, to prove the existence of the desired sequence of best responses, it is sufficient to prove it for networks where at least one player receives all the information without loss. The first algorithm takes one such network as its input and a wheel of trees as its output such that all nodes except perhaps those in the central wheel are playing their only best responses.

Algorithm 1: The algorithm involves the following variables: the current network $g$, a current reference node $v$ s.t. $I_{v}(g)=n-1$, a partition of $N$ into two sets of nodes: $W$ (nodes forming an oriented line rooted at $v$ ), $V=N \backslash W$, and a subset $V^{*} \subseteq V$ of nodes already tested.

START (Initialization): Let $g^{0}$ be the initial network and $i_{0}$ a player such that $I_{i_{0}}\left(g^{0}\right)=n-1$, then make $g:=g_{0}, v:=i_{0}, W:=\varnothing, V:=N$ and $V^{*}:=\varnothing$.

Step 1: If $V \neq V^{*}$, apply the Selection Procedure (see below) to select a node $j \in V \backslash V^{*}$ for testing and go to step 2. If $V=V^{*}$, END.

Step 2 (Test at node $j$ w.r.t. reference node $v$ ): Does $j$ have a best response in which $\overleftarrow{j v}$ is one of the links?:

- If Yes, then let $j$ play a best response where link $\overleftarrow{j v}$ is part of and sever all links that do not disconnect the network. Then make $g$ equal to the resulting network, make $v:=j$ (new reference node), $W:=W \cup\{j\}, V:=N \backslash W$, and $V^{*}:=\varnothing$ and go to Step 1. 


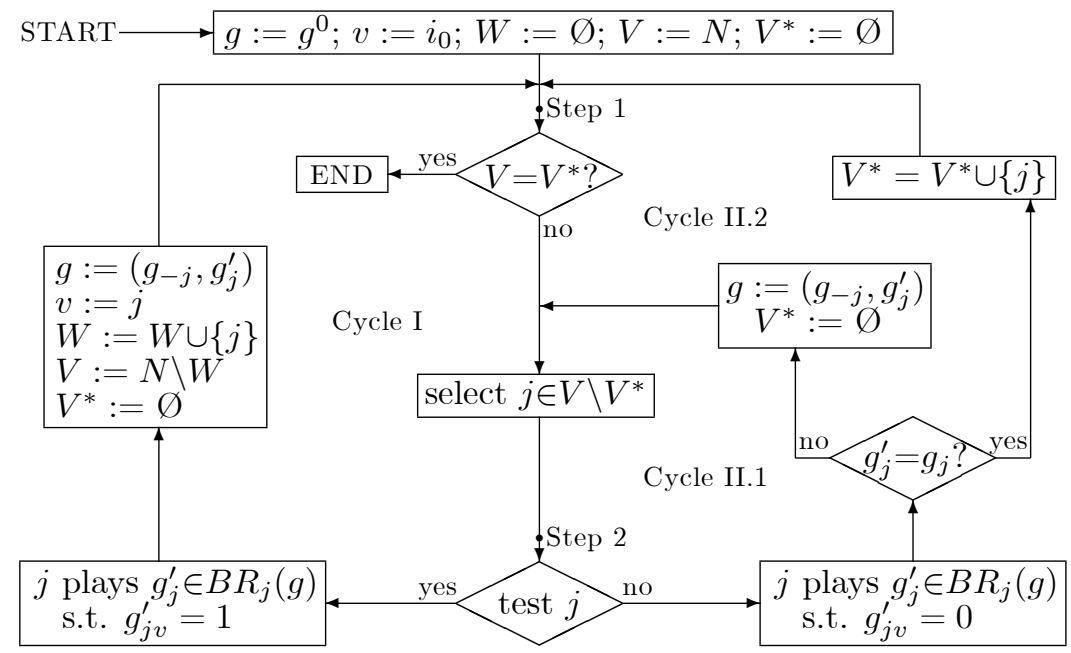

Figure 8: Algorithm 1

- If $N o$, then let $j$ play a best response consisting of deleting all its links that do not disconnect the network, with two possible continuations:

- If $j$ 's best response to $g$ is $g_{j}$ (i.e. $j$ does nothing) then make $V^{*}:=$ $V^{*} \cup\{j\}$ and go to Step 1 .

- If $j$ 's best response to $g$ is other than $g_{j}$ (i.e. $j$ severs one or more links) then make $g$ equal to the resulting network and $V^{*}:=\varnothing$ and go to Step 1 (i.e. to the Selection Procedure given that $V \neq V^{*}=\varnothing$ ).

Figure 8 represents the diagram of the algorithm, where $B R_{j}(g)$ denotes the set of best responses of node $j$ to $g_{-j}$.

Selection Procedure (Selection of a node $j$ for testing w.r.t. $v$ ): Let $g$ be the current network, let $v$ be the current reference node and $V, W \subseteq N(V \neq \varnothing, W \neq N)$ and $V^{*} \neq V$ the current sets. Select a node $j$ among those in $V \backslash V^{*}$ for which the length of the shortest $v$-oriented path to $v$ in $g$ is the largest.

Let us first see that the test at node $j$ is sound in the sense that it yields only one of the two outcomes specified.

Claim 1 Let $v$ be the current reference node, and let $j \in V \backslash V^{*}$ be the node for testing w.r.t. $v$ selected by the Selection Procedure. Then $j$ either has a best response where s/he links node $v$ and severs all other links as long as they do not disconnect the network, or a best response of $j$ consists of just deleting all links that do not disconnect (weakly) the network. ${ }^{17}$

\footnotetext{
${ }^{17}$ Note that in both cases such a best response may coincide with $j$ 's current strategy, i.e. with $g_{j}$. In this case the network remains unchanged but the process continues.
} 
Proof. First note that, since $c<1$, a best response of $j$ cannot sever the links that would disconnect the network. Assume that $j$ has a best response to $g_{-j}$ where $j$ links at least one node $k$ and this link is not necessary to keep the network connected. If $g^{\prime}$ is the network that results by playing such a best response, then $\Pi_{j}\left(g^{\prime}\right) \geq \Pi_{j}(g)$. Since $I_{v}(g)=n-1$ no link can give $j$ a greater payoff than linking with $v$. Therefore $j$ has a best response where $j$ only supports a link with node $v$ in addition to those whose deletion would disconnect the network. Any other link would be superfluous.

Now let us check that in either case the best response of the node tested cannot damage the reference node's information and when the node tested becomes the new reference node it keeps receiving information $n-1$.

Claim 2 After completion of either Cycle I or Cycle II (via II.1 or II.2) the (same or new) reference node keeps receiving information $n-1$ from the network.

Proof. Let $v$ be the current reference node, let $j \in V \backslash V^{*}$ be the node selected for the test by the Selection Procedure, and let $g$ be the current network and $V^{*}, V, W$ the current sets of nodes. That is, $j$ is a node among those in $V \backslash V^{*}$ for which the length of the shortest $v$-oriented path to $v$ is the largest. In order to prove that a best response of $j$ cannot damage $v$ 's information it is enough to check that if deletion is part of a best response of $j$ for any link $\overleftarrow{j j^{\prime}}$ that $j$ supports in $g$ then $I_{v}(g)=I_{v}\left(g-j j^{\prime}\right)$. Three cases can be distinguished:

Case 1: $j^{\prime} \in V \backslash V^{*}$. Then, given the choice of $j$, the shortest $v$-oriented path from $j^{\prime}$ to $v$ must necessarily be shorter than or of the same length as that from $j$ to $v$. In other words, a $v$-oriented path from $j^{\prime}$ to $v$ exists that does not use the link $\overleftarrow{j j^{\prime}}$ and consequently its deletion would not cause any loss of information to $v$.

Case 2: $j^{\prime} \in W$. In this case, by construction, $v$ receives all the information from $j^{\prime}$ via a $v$-oriented path within $W$ so that deleting $\overleftarrow{j j^{\prime}}$ leaves that path intact.

Case 3: $j^{\prime} \in V^{*}$. This may only occur after a completion of Cycle II.2, and in this case $j^{\prime}$ supports only those links whose deletion would cause the disconnection of the network. In this case a best response of $j$ would not delete link $\overleftarrow{j j^{\prime}}$ because this would disconnect the network and cause a loss to $j$.

Thus no deletion that is part of a best response of $j$ can damage $v$ 's information. Therefore, after the test at $j$, in the case of "No" (i.e. Cycle II), where the reference node remains the same, the reference node keeps its information intact. In the case of "Yes" (i.e. Cycle I), j's best response includes link $\overleftarrow{j v}$ and, as $v$ keeps its information intact, $j$, the new reference node, also receives $n-1$ in the new network.

Finally, let us see that the algorithm always terminates as desired.

Claim 3 Algorithm 1 terminates after a finite number of iterations and at the end yields a wheel of trees where all nodes except perhaps those in the central wheel are playing their only best responses. 
Proof. The algorithm involves two cycles. After a completion of Cycle I, $W$ contains a new node and $V$ one less. As to Cycle II, note that after each completion via II.2 $V^{*}$ contains one more node. Note also that each iteration via Cycle II.1 involves deleting some links, which means that after a finite number of iterations the process must necessarily continue either through Cycle I or Cycle II.2. Thus, in finite time $N=W \cup V^{*}$ and $V=V^{*}$, and the algorithm ends. At this point either $V=V^{*}=N$ so that $W=\varnothing$, or $W \neq \varnothing$. Let us discuss the two cases:

- Case $W=\varnothing$ : this case occurs when Cycle I is never carried out. Then the resulting network is an oriented tree whose root is the initial node $i_{0}$, and where no terminal node improves or equals its payoff by creating a link with the root. In other words this oriented tree is a strict Nash network.

- Case $W \neq \varnothing$ : in this case, by construction, when the algorithm ends nodes in $W$ form either an oriented line or a wheel, and those in $V=V^{*}$ form root-oriented trees rooted in $W$. Thus, in the first case (nodes in $W$ form an oriented line) the final network is an oriented tree, in the second (nodes in $W$ form an oriented wheel) the final network is a wheel of trees. In both cases creating a new link with any node in $W$ is not a best response for nodes in $V=V^{*}$. For those in $W$, two cases must be distinguished. If they form an oriented line, note that the last node in this line is the first node joining $W$, which maintains at least one link with the initial one $i_{0}$. In this case the process ends with $i_{0} \in V^{*}$. Therefore creating a link with the root is not a best response for any of the terminal nodes of the final oriented tree, and consequently it is a strict Nash network. If the nodes in $W$ form an oriented wheel, then for all other nodes in the tributary trees linking with the central wheel is not a best response.

Therefore when the sequence of best responses generated by Algorithm 1 yields an oriented tree it is certain to be a strict Nash network and we are done. However when the output is not a tree, i.e. it is a wheel of trees, it may be that a node in the central wheel is interested in breaking it. In view of this possibility, the second algorithm takes as its input a wheel of trees where all nodes except perhaps those in the central wheel are playing their only best responses.

Algorithm 2: The algorithm involves the following variables: the current network (a wheel of trees or an oriented tree) $g$, a current reference node $v$ s.t. $I_{v}(g)=n-1$, a set of nodes $W$ (forming the current central wheel), and a subset $W^{*} \subseteq W$ of nodes already tested.

START (Initialization): Let $g^{0}$ be the initial network (a wheel of trees) and $i_{0}$ a player in the central wheel, then make $g:=g^{0}, v:=i_{0}$, let $W$ the set of players in the central wheel in $g^{0}$ and let $W^{*}=\varnothing$.

Step 1: If $W \neq W^{*}$, go to Step 2. If $W=W^{*}$, END-1 ( $g$ is a strict Nash wheel of trees).

Step 2: Is breaking the central wheel a best response of $v$ ?

- If $N o$, then make $W^{*}:=W^{*} \cup\{v\}$ and take a new node $j$ in the central wheel, i.e any $j \in W \backslash W^{*}$, make $v:=j$ and go to Step 1 . 


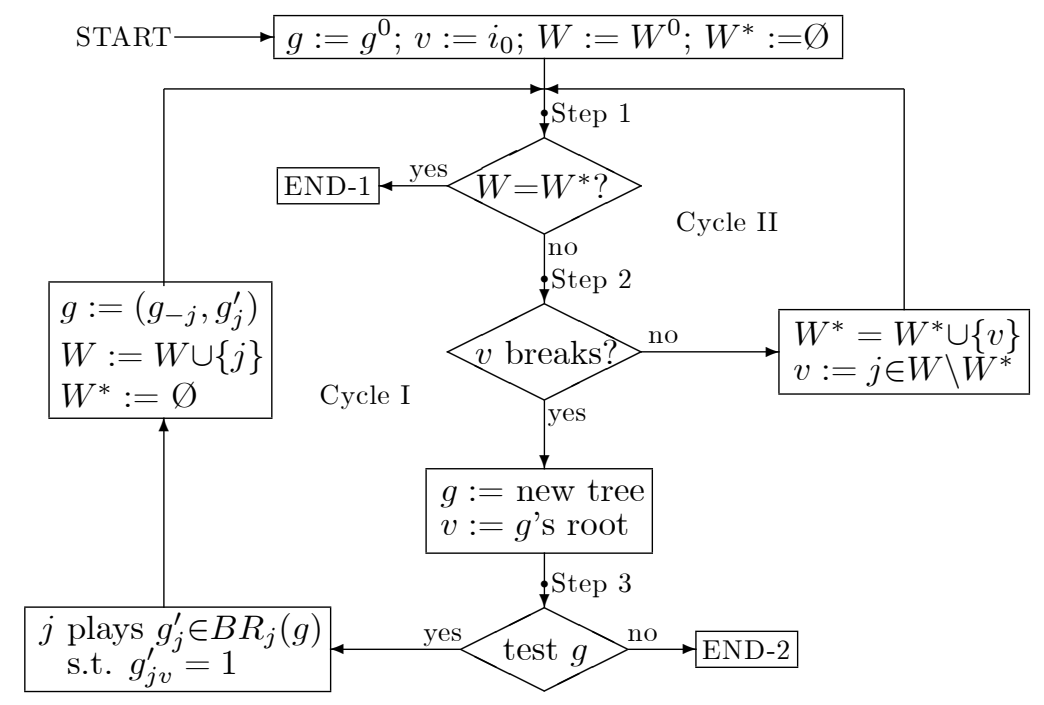

Figure 9: Algorithm 2

- If Yes, then let $v$ break the central wheel, update $g$ as the resulting oriented tree, and let $v$ be the root of the tree and go to Step 3.

Step 3: Is linking with the root $v$ a best response of a furthest (from $v$ ) terminal node $j$ ?

- If No, END-2 ( $g$ is a strict Nash oriented tree).

- If Yes, then let $j$ link with the root $v$, update $g$ as the resulting wheel of trees, let $W:=W \cup\{j\}$ be the new central wheel and $W^{*}=\varnothing$, and go to Step 1 .

Figure 9 represents the diagram of the algorithm.

Claim 4 At END-1 the output of Algorithm 2 is a strict Nash wheel of trees, and at END-2 it is a strict Nash oriented tree.

Proof. First note that at Step $1 g$ is a wheel of trees where all nodes except perhaps those in the central wheel are playing their only best responses. This is so at the start and also after any completion of cycles I and II. For Cycle II this is obvious since the network remains unchanged. In Cycle I Step 1 is reached after a node breaks the central wheel and the furthest node away from the root of the resulting tree forms a new central wheel by linking with the root. Therefore, in the new wheel of trees terminal nodes cannot be at a greater discounted distance from the central wheel than in the preceding one. Thus, at Step $1 g$ is a wheel of trees where all nodes except perhaps those in the central wheel are playing their only best responses. END-1 is reached after checking that breaking the central wheel is not a best response for any node in it. Therefore, at END-1 the output is a strict Nash wheel of trees. 
As to END-2, note that at Step $3 g$ is an oriented tree that is the result of breaking a wheel of trees. Therefore its architecture is that of an oriented line some of whose nodes support some links. For this particular architecture of an oriented tree it can be checked straightforwardly that condition (6) holds for all terminal nodes if and only if it holds for those furthest away from the root. In other words, this is necessary and sufficient for the tree to be a strict Nash network. Therefore at END-2 the output is a strict Nash oriented tree.

Thus, in both cases the output is a strict Nash network. Therefore, only remains to prove that the algorithm necessarily terminates in finite time.

Claim 5 When its input is a wheel of trees where all nodes except perhaps those in the central wheel are playing their only best responses, Algorithm 2 terminates generically in finite time.

Proof. There are two cycles. Cycle II cannot be repeated more times than there are nodes in $W$. As to Cycle I, it may be the case that a node in $W$ is indifferent between keeping and severing its link in $W$. For instance, within the curve $c=n-1-\alpha-\alpha^{2}-$ $\ldots-\alpha^{n-1}$ this situation occurs: the oriented wheel and the oriented line are Nash but not strict Nash. More generally, this occurs when the right-hand side of (8) holds in the form of equality for the cyclic permutation for which this condition is most demanding, that is, when

$$
c=\min _{p}\left(n-\left(w_{p\left(i_{1}\right)}+w_{p\left(i_{2}\right)} \alpha+\ldots+w_{p\left(i_{k}\right)} \alpha^{k-1}\right)\right) .
$$

But this may only occur for a subset of values of the parameters of zero measure. Otherwise every time Cycle I is done a new wheel of trees is formed with one more link in the central wheel than the preceding one, which sets an obvious bound to the number of possible repetitions of this cycle.

Remarks: (i) Observe that inertia has played an implicit role, as it has been implicitly assumed that at each best response in the sequence all other players keep their strategies, but miscoordination has played no role. In other words, the result remains the same assuming a different dynamic model without inertia, where in every period only one player plays a best response. ${ }^{18}$

(ii) The algorithms can be refined in some points, but given that they are intended to provide proof rather than for operational purposes, priority is given to simplicity over computational efficiency. ${ }^{19}$

\footnotetext{
${ }^{18}$ Nevertheless, in the curve where both the wheel and the line are Nash but not strict Nash networks, a miscoordination move in the wheel where all nodes delete their only link followed by a best response of a single node would yield a center-sponsored star which is strict Nash throughout the curve.

${ }^{19}$ For instance, in Cycle I in Algorithm 1, when $g_{j}^{\prime}=g_{j}$ (i.e. the best response of the player tested coincides with the current one) it is unnecessary to reset $V^{*}:=\varnothing$.
} 


\section{Conclusions}

We provide a new model of non-cooperative network formation with asymmetric flow. Unlike other contexts where asymmetry is just the result of dropping a symmetry assumption, often with the purpose of obtaining a better understanding of the implications of symmetry, here asymmetry seems a natural assumption. Moreover, this model bridges the gap between two benchmark models so far seen as "orthogonal", and gives rise to a significant variety of stable architectures. The beauty and elegance of Bala and Goyal's results for the two benchmark models, yielding as strict Nash networks two primary and emblematic architectures, the wheel and the star, gives rise at the same time to a certain uneasiness as one feels that other structures should also arise from simple assumptions. It turns out that just a little asymmetry opens the door to another remarkable structure, hierarchical root-oriented trees, as strictly stable, and also, within a margin for the parameters, the interesting wheels of trees, which are midway between the wheel and the star and include both as particular cases.

From the point of view provided by the asymmetric flow model, the two-way flow model appears as an extreme and somehow pathological case: in a center-sponsored star one player pays everything and everybody sees everything, while in the asymmetric flow model in a center-sponsored star the center pays everything but receives more information than anybody else, and in other stable architectures players receive more or less information depending on their position. Thus stability and asymmetry yield a clear hierarchy from the point of view of the amount of information received. In both Nash and strict Nash architectures, one set of players, the root in the tree, the central wheel in the wheel of trees or the maze in a Nash network, receives all the information intact from the network, while the rest, plugged in to tributary trees, receive it with a certain decay. It is remarkable how such simple premises yield in equilibrium these somewhat zoomorphic forms, which embody logic from the informational point of view and appear as meaningful information-processing proto-structures.

As to dynamics, a smooth extension of Bala and Goyal's results has also been possible, although the greater complexity of the new setting has required a lengthy algorithmic constructive proof.

The model opens up several lines for further research. First, all the extensions explored in the literature for either of the two benchmark models of Bala and Goyal can be studied taking the asymmetric flow model as point of departure. Second, note that if a certain decay $\beta \geq \alpha$ is also assumed in the flow through a link towards the player that supports it, then the model includes also as particular cases the one-way and two-way flow models with decay. Namely, in this model with "asymmetric decay", the case $0<\beta<1$ and $\alpha=0$ is the one-way flow model with decay, while the case $0<\alpha=\beta<1$ is the two-way flow model with decay. Here again the two-parameter ( $\alpha$ and $\beta$ ) model covers all intermediate situations between the two models. As to dynamics, footnote 18 suggests the possibility of a stronger version of Theorem 4. Still within the framework of the model considered here, it may be of interest to introduce 
mistakes in the dynamic model in order to study the stochastic stability of strict equilibria. $^{20}$ Finally, it may also be interesting to examine the effects of asymmetric flow on models based on pairwise stability à la Jackson and Wolinsky.

\section{References}

[1] Bala, V., and S. Goyal, 2000a, A noncooperative model of network formation, Econometrica 68, 1181-1229.

[2] Bala, V., and S. Goyal, 2000b, A strategic analysis of network reliability, Review of Economic Design 5, 205-228.

[3] Billand, P., C. Bravard, S. Sarangi, 2008, Existence of Nash networks in one-way flow models, Economic Theory, 37, 491-507.

[4] Bloch, F., and B. Dutta, 2009, Communication networks with endogenous link strength, Games and Economic Behavior 66, 39-56.

[5] Derks, J., Kuipers, J., Tennekes, M., Thuijsman, F., 2009, Existence of Nash networks in the one-way flow model of network formation. In: Neogy, S.K. et al. (eds.) Modeling, Computation and Optimization, World Scientific.

[6] Derks, J., M. Tennekes, 2009, A note on the existence of Nash networks in one-way flow models, Economic Theory, 41, 515-522.

[7] Feri, F., 2007, Stochastic stability in networks with decay, Journal of Economic Theory 135, 442-457

[8] Galeotti, A., 2006, One-way flow networks: the role of heterogeneity, Economic Theory 29, 163-179.

[9] Galeotti, A., S. Goyal and J. Kamphorst, 2006, Network formation with heterogeneous players, Games and Economic Behavior 54, 353-372.

[10] Goyal, S., 2007, Connections. An Introduction to the Economics of Networks, Princeton University Press. Princeton.

[11] Goyal, S., and F. Vega-Redondo, 2005, Network formation and social coordination, Games and Economic Behavior 50, 178-207.

[12] Hojman, D.A., and A. Szeidl, 2008, Core and periphery in networks, Journal of Economic Theory 139, 295-309.

\footnotetext{
${ }^{20}$ See Feri (2007), also Jackson and Watts (2002).
} 
[13] Jackson, M., 2008, Social and Economic Networks, Princeton University Press. Princeton.

[14] Jackson, M., 2010, An overview of social networks and their analysis, in The Handbook of Social Economics, edited by J. Benhabib, A. Bisin, and M.O. Jackson, Elsevier Press.

[15] Jackson, M., and A. Watts, 2002, The evolution of social and economic networks, Journal of Economic Theory 106, 265-295.

[16] Jackson, M., and A. Wolinsky, 1996, A strategic model of social and economic networks, Journal of Economic Theory 71, 44-74.

[17] McBride, M., 2006, Imperfect monitoring in communication networks, Journal of Economic Theory 126, 97-119.

[18] Olaizola, N., and F. Valenciano, Network formation under institutional constraints, http://www.bridgebilbao.es/archivo/ficheros/federico/il5111revised.pdf.

[19] Olaizola, N., and F. Valenciano, One-way network formation under constraints, http://www.bridgebilbao.es/archivo/ficheros/norma/il5812.pdf.

[20] Vega-Redondo, F., 2007, Complex Social Networks, Econometric Society Monographs, Cambridge University Press. 